\title{
Efectos del juego en el estado de ánimo en jóvenes y adultos: estudio preliminar
}

Effects of play on mood in youth and adults: preliminary study

Recepción del artículo: 30-12-2019 | Aceptación del artículo: 26-04-2020

Mariana La Valle Universidad Abierta Interamericana - Facultad de Psicología y Humanidades (Argentina) mariana_1v5@yahoo.com.ar

https://orcid.org/0000-0002-4318-0238

Alba Elizabeth Mustaca Universidad Abierta Interamericana - Facultad de Psicología y Humanidades Centro de Altos Estudios en Ciencias Humanas y de la Salud (CAECIHS) (Argentina) albamustaca@gmail.com

https://orcid.org/0000-0001-9699-3147

\section{Resumen}

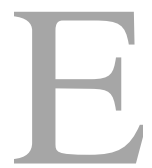

sta investigación tuvo como objetivo evaluar los efectos de juegos de salón en el estado de ánimo. Se realizó un diseño cuantitativo, cuasi-experimental, intrasujeto. Participaron 10 voluntarios de entre 15 y 65 años en dos almuerzos con un intervalo de un mes entre ellos. Se aplicó la Escala de Valoración del Estado de Ánimo (EVEA) antes y después de cada reunión. El primer almuerzo (sin juego) finalizó después de la sobremesa; el segundo (con juego) se realizaron juegos de cartas y de lectura y escritura. En ambos grupos se halló que la ansiedad disminuyó significativamente después del almuerzo $(\mathrm{p}<0.01, \mathrm{r}=.71)$. En el almuerzo con juego aumentó la alegría $(\mathrm{p}<0.01, \mathrm{r}=1)$ y disminuyó la ira y la hostilidad $(\mathrm{p}<0.02, \mathrm{r}=1)$ y la tristeza y depresión $(\mathrm{p}<0.05, \quad \mathrm{r}=.71)$. Se deben realizar futuras investigaciones para corroborar este hallazgo y evaluar otros efectos, tanto emocionales como cognitivos y su duración.

Palabras clave: Juego, estado de ánimo, adultos.
Para referenciar este artículo:

La Valle, M. y Mustaca, A. (2020). Efectos del juego en el estado de ánimo en jóvenes y adultos: estudio preliminar. Revista ConCiencia EPG, 5(1), 38-50. https://doi.org/10.32654/CONCIENCIAEPG. 


\section{Abstract}

his research aimed to evaluate the effects of board games on mood. A quantitative, quasi-experimental, intrasubject design was performed.

Ten volunteers between 15 and 65 participated in two lunches with an interval of one month between them. The Mood Assessment Rating Scale (EVEA) was applied before and after each meeting. The first lunch (without game) ended after the table; the second (with game) card and reading and writing games were made. In both groups it was found that anxiety decreased significantly after lunch $(\mathrm{p}<0.01, \mathrm{r}$ $=.71)$. At lunch with play, joy increased $(\mathrm{p}$ $<0.01, r=1)$ and decreased anger and hostility $(\mathrm{p}<0.02, \mathrm{r}=1)$, and sadness and depression ( $\mathrm{p}$ $<0.05, r=.71)$. Future research should be carried out to corroborate this finding and evaluate other effects, both emotional and cognitive and its duration.

Key words: Game, mood, adults.

\section{Introducción}

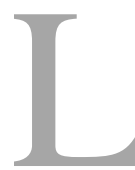

a palabra juego procede de la palabra latina iocus, que significa diversión y bromas. Se define como cualquier actividad libre que se realiza con el fin de divertirse, generalmente siguiendo determinadas reglas. Cabe señalar que el juego y el deporte son actividades muy parecidas: en ambos se aplican habilidades motoras, intelectuales y sociales, siguiendo una serie de reglas; la diferencia reside que en el deporte el objetivo principal es competir para comparar capacidades (Velásquez, 2014) y en el juego, es entretenerse.

En los niños la función del juego fue investigado por psicólogos, etólogos, sociólogos, etc. aunque está incorporado en todas las etapas evolutivas del ser humano; tanto los niños como los adultos de todas las épocas han jugado. Notablemente existen escasos estudios experimentales o cuasi experimentales que muestren sus efectos en adultos, probablemente debido a que intuitivamente está consensuado en la mayoría de las culturas que provoca bienestar y salud en todos sus niveles y a las dificultades para realizar estudios longitudinales controlados. Sin embargo, cada vez más se los utilizan como herramientas de aprendizaje, rehabilitación y distintos objetivos culturales, educativos y terapéuticos.

En adultos mayores se evaluaron los efectos del uso juegos digitales como herramientas para el entrenamiento físico y cognitivo. Estos juegos requieren progresivamente mayor precisión, mayor velocidad y la supresión de información irrelevante, lo que puede conducir cambios neurológicos positivos en los sistemas cerebrales. Además, en la mayoría de los juegos digitales se requiere localizar rápidamente un lugar adecuado en la pantalla, coordinación mano-ojo y atención sostenida a la tarea. A medida que los jugadores practican y se vuelven competentes en estas tareas, se espera que se desarrollen sus habilidades visuales-espaciales. Además, los juegos digitales son agradables de usar como herramientas para el entrenamiento físico y cognitivo porque están diseñados para ser divertidos de jugar. Por lo general tienen recompensas extrínsecas como nivel, armas elegantes y pantallas de puntaje alto que 
motivan a los jugadores a continuar frente a los desafíos. Los jugadores estarán motivados para completar tareas y serán llevados al límite de sus habilidades sin asustarse de la complejidad de la tarea. Además, con los avances de la tecnología de realidad virtual, los juegos digitales somatosensoriales que combinan juegos digitales tradicionales y actividades físicas se convierten en una innovación para proporcionar oportunidades de ocio alternativas para adultos mayores; estos juegos pueden recibir comentarios, señales, detectar los gestos de los jugadores y movimientos corporales para interactuar con los juegos digitales. Por ejemplo, Wii Fit incluye Más de 40 actividades (posturas de yoga y entrenamiento de fuerza, etc.) diseñadas para que el jugador haga ejercicio físico. Los jugadores tienen que hacer cambios inmediatos, imprevistos y en múltiples direcciones a través de ajustes posturales en una tabla de equilibrio en respuesta a tareas visuales orientadas a objetivos. El último metaanálisis sobre los efectos de juegos digitales que incluyen a los tradicionales como a los somatosensoriales con actividad física, (ej. Nintendo Wii Fit, XBOX 360 lanzado por Microsoft y Play Station Eye publicado por Sony) fue realizado por Zhang y Kaufman (2016). Presentaron 5 metaanálisis sobre 36 estudios publicados en 2012 y 2013. Hallaron que esos juegos tienen efectos beneficiosos sobre el equilibrio, la movilidad, la función ejecutiva y la velocidad de procesamiento de los adultos mayores que viven en la comunidad y en hogares de ancianos; y que la correlación entre esos efectos, las edades de los participantes y el tiempo de uso arrojó un tamaño del efecto débil.

Los juegos digitales también se utilizaron como herramientas para el manejo de enfermedades y adherencia a diferentes tratamientos. Perandones Serrano (2010) menciona recientes investigaciones que persiguen como fin algún tipo de beneficio o información sobre la salud de las personas. Estos videojuegos se diseñan para sujetos que están enfermos. La finalidad es enseñarles los procesos que se están llevando a cabo en de su cuerpo y los mejores hábitos que pueden adoptar frente a la enfermedad, en reducir el estrés o la ansiedad derivados de los tratamientos y permitirles exteriorizar las ganas de curarse y de luchar y desmitificar a las enfermedades, convirtiéndolas en algo más natural y menos monstruoso de lo que a menudo parecen, para mejorar no sólo su aceptación sino también la no-estigmatización social de los pacientes. Se halló un solo estudio experimental sobre el uso de videojuegos en el ámbito hospitalario y de salud con el videojuego Re-Mission. Kato, Cole, Bradlyn y Pollock (2008) realizaron un diseño aleatorio con grupo control cuyo propósito fue determinar la efectividad de una intervención del videojuego para mejorar la adherencia y otros resultados conductuales para adolescentes y adultos con neoplasias, incluyendo leucemia aguda, linfoma y sarcoma de tejidos blandos. Se realizó un ensayo aleatorio con evaluaciones iniciales y de 1 y 3 meses entre 2004 y 2005 en 34 centros médicos en los Estados Unidos, Canadá y Australia. Se evaluaron un total de 375 pacientes masculinos y femeninos que tenían entre 13 y 29 años con un diagnóstico inicial o de recaída de una neoplasia maligna, que se encontraban en tratamiento y se esperaba que lo continuaran durante al menos 4 meses desde la evaluación inicial. Se asignaron aleatoriamente a la intervención o grupo control. La intervención fue un videojuego que abordó cuestiones de tratamiento y atención del cáncer para adolescentes y adultos jóvenes. Las medidas dependientes incluyeron 
adherencia al tratamiento de antibióticos y otros medicamentos, autoeficacia, conocimiento de la enfermedad, control, estrés y calidad de vida. Los resultados mostraron que la adherencia a la toma de medicación fue mayor en el grupo de intervención al igual que la autoeficacia y el conocimiento de la enfermedad en comparación con el grupo control. Sin embargo, el uso de videos juegos no afectaron las medidas subjetivas de autoinformes de adherencia, estrés, control y calidad de vida.

Respecto de los juegos de salón y de creatividad, Linares Cervantes (2015) realizó un estudio cualitativo que utilizó el juego de creatividad de mesa ESPABILA elaborado con base en las técnicas creativas de ideogramación, brainstorming, matriz de descubrimiento y check list con grupos de inmigrantes de España con el objetivo de aumentar su integración a la cultura española. Sus resultados tomando entrevistas y grupos focales indicaron que los participantes presentaron reacciones socio-emotivas positivas, fueron solidarios, prestaron ayuda a los demás, valoraron la participación de los otros, propició distensión de los inmigrantes; mostraron un alto grado de satisfacción, expresado con sonrisas y bromas y muy pocos participantes "abandonaron mentalmente" el juego.

Entre los juegos de mesa, el ajedrez fue uno de los preferidos para su investigación. Como estudios pioneros cabe destacar los trabajos de De Groot (1946, 1965), de Chase y Simon (1973) y de Gobet y Simon (1996, 1998). Ya en el s. XXI, los de Linhares y colaboradores (Linhares, 2005; Linhares \& Freitas, 2010; Linhares, Freitas, Mendes, \& Silva,( 2012), Charness, Tuffiash, \& Krampe (2005), Bilalic', Langner, Erb, \& Groud
(2010); Bilalic', McLeod, \& Gobet, (2009) y Campitelli, Gobet y Parker (2005) entre otros. Estos estudios en su mayoría se llevaron a cabo con población adulta, comparando jugadores de ajedrez expertos frente a novatos. En sus resultados se constató la superioridad de los ajedrecistas expertos en aquellas tareas cognitivas más estrechamente relacionadas con el juego de ajedrez. Los trabajos más recientes incorporan, mediante resonancia magnética funcional, el análisis de los mecanismos y las bases neuronales del reconocimiento de objetos y patrones (e.g. Campitelli et al., 2005; Gobet, \& Campitelli, 2007). Hernández y Rodríguez (2006) presentaron evidencia de cómo los jugadores de ajedrez con más éxito utilizan modos de valorar y afrontar la realidad más realistas, positivos y ponderados. Otros resultados más controlados indican que los ajedrecistas desarrollan capacidades específicas para el juego de ajedrez. Por ejemplo, aumentan la memorización de las posiciones de las fichas si son las usadas comúnmente, pero si se le presentan posiciones al azar, su memorización no difiere de los participantes que no saben jugar al ajedrez (Campitelli, 2017, comunicación personal).

En un trabajo cuasiexperimental realizado para evaluar el uso del ajedrez en la educación de los niños, Aciego, García y Betancort (2016) dividieron en 3 grupos a niños y adolescentes de entre 6 y 16 años. El grupo ajedrez 1 tuvo una formación integral que desarrolló una metodología basada en el aprendizaje significativo, implicaba la participación de todos los alumnos y el trabajo de capacidades cognitivas y emocionales; su objetivo principal era que se disfrute con el ajedrez, que se apasione y sienta deseos de aprender. Hacíoan ejercicios de lengua como sopas de letras, jeroglíficos, autodefinidos, 
lecturas, cuentos, ejercicios de matemáticas, como jugar a los barquitos (para el aprendizaje de la notación algebraica y descriptiva) y problemas sumatorios de valores de las piezas, concursos por equipos, en los que se responde a series de preguntas relacionadas con conocimientos generales y ajedrecísticos, clases de tutores, que consistía en dar a los alumnos/as responsabilidades sobre sus compañeros/ as, de manera que además de enseñarles contenidos a los miembros de su grupo (que el monitor previamente les había enseñado a ellos), debían hacer que su grupo cumpla las normas de convivencia y de trabajo planteadas desde principio de curso. El grupo ajedrez 2, consistió en la formación centrada en el tablero $(n=60)$; y grupo de comparación 3 practicó baloncesto y fútbol $(n=60)$. Se midieron competencias cognitivas, evaluada mediante prueba de rendimiento; socioafectivas, mediante tests auto-evaluativos; y en ajedrez, mediante prueba de rendimiento. Se obtuvo que el grupo de formación integral mejoró no solo en competencias cognitivas básicas (atención y memoria), como ocurre en el grupo 2, sino también en competencias cognitivas más complejas (asociación, análisis y síntesis, planeamiento y previsión, entre otras). Igualmente mejoraron en el ámbito socio personal no solo en el comportamiento en clase, sino también en autoestima, motivación y actitud ante el estudio comparado con el grupo control. Es de notar que en este estudio lo que muestra es la superioridad de una educación integral, el valor del aprendizaje del ajedrez se confunde con la intervención; el grupo 2 sería el que aporta el resultado que hallo una mejora en atención y memoria.

El trabajo de Carrasco, Zumba y Cuenca Quito (2015) tuvo como objetivo diseñar y aplicar un programa de aprendizaje y práctica del ajedrez para los jubilados que participan en el Centro de atención al adulto mayor del Instituto Ecuatoriano de Seguridad Social "IESS" de la ciudad de Cuenca con la realización de un taller de ajedrez durante el período febrero a mayo de 2015.El propósito era mejorar la calidad de vida de los participantes, aprovechando los beneficios de la práctica del ajedrez, sobre todo en sus capacidades intelectuales y para promoverla como una de las actividades recreativas para los adultos mayores. Se utilizó un procedimiento experimental de un grupo Pre post test usando la evaluación cognitiva Montreal (MoCA) y otra sobre conocimiento del ajedrez en una muestra de 8 personas. Los resultados mostraron que los participantes tuvieron un aumento de las capacidades cognitivas evaluadas; sin embargo, el artículo no presentó los análisis de significación estadística por lo cual no se logra saber si el ajedrez modifica realmente las funciones cognitivas en esa población.

En adultos mayores se utilizan los juegos salón como estimulación cognitiva e integración social. Noda, Shirotsuki y Nakao (2019) presentaron una revisión sistemática y metaanálisis sobre sus efectos en la educación y en su calidad de vida. Entre enero de 2012 y agosto de 2018 identificaron 83 artículos; 56 (67\%) de educación o entrenamiento dirigido para problemas relacionados con la salud, seis (7\%) examinaron los mecanismos cerebrales básicos, cinco (6\%) evaluaron medidas preventivas para la demencia o contribuciones al envejecimiento saludable, y tres (4\%) evaluaron la comunicación social o políticas de salud pública. Los resultados de un número limitado de ensayos controlados aleatorizados indicaron que jugar juegos de mesa tradicionales (por ejemplo, ajedrez, Go, y Shogi) ayuda a mejorar el deterioro cognitivo 
y la depresión, y otros juegos de mesa recientemente desarrollados son beneficiosos para la promoción de una alimentación saludable, dejar de fumar y tener relaciones sexuales seguras. Además, proporcionaron hallazgos sobre la función cerebral, los efectos cognitivos y la modificación de los factores relacionados con el estilo de vida. Aunque son muy promisorios, estos resultados tienen limitaciones. La mayoría de ellos son estudios de un solo grupo evaluados antes y después de la intervención, hay escasos trabajos con un grupo controles sin juego y ninguno con un tratamiento placebo, y los tamaños de la muestra fueron bajos.

En conclusión, los estudios presentados son promisorios en cuanto a los efectos cognitivos, físicos y emocionales de jugar en adultos mayores, enfermos, en los juegos de salón los más estudiados fueron los efectos del aprender ajedrez, y se hallaron pocas investigaciones experimentales o cuasiexperimentales que confirmaran las hipótesis planteadas.

La presencia de juegos en las reuniones infantiles es casi una obligación. En las reuniones de adultos, en cambio, predominan las conversaciones y tertulias. Otra de las funciones de los juegos en adultos sanos puede ser disminuir el estrés y mejorar el estado de ánimo, integrar distintas edades en reuniones familiares y aumentar las emociones positivas. ¿Si se introducen juegos en las reuniones de adultos, mejorará más el estado de ánimo de los participantes comparado con las reuniones donde se conversa? La siguiente investigación tuvo como objetivo general evaluar si el juego mejora el estado de ánimo en una reunión de amigos y familiares. Para ello se comparó el estado de ánimo de un grupo de jóvenes y adultos antes y después de dos almuerzos donde en uno de ellos se incluyeron juegos de creatividad. Se realizó un diseño cuasiexperimental intrasujeto pre- post test, con una variable independiente activa: juegos de creatividad, con dos valores: $\mathrm{Si}$ - No y una variable dependiente: estado de ánimo medido con la Escala de Valoración del Estado de Ánimo (EVEA) antes y después de la manipulación experimental. Como variables extrañas controladas fueron similitud en el lugar y el almuerzo en las dos condiciones experimentales, las diferencias individuales y que en la condición control (sin juegos creativos) la sobremesa fue de más duración que en la condición de juego, para que el tiempo de contacto social del grupo sea relativamente igual en los dos encuentros y la conversación sea un control más adecuado. Se predice que la introducción de juegos después de un almuerzo mejorará más el estado de ánimo de los participantes que una mera sobremesa.

\section{Método}

Participantes. Se tomó una muestra no aleatoria y voluntaria de la Ciudad Autónoma de Buenos Aires y de la Provincia de Bs. As. que eran parientes y amigos de la primera autora. Estuvo formada por 4 varones de 14, 17,28 y 39 años y 6 mujeres de 15, 21, 36, 42, 43 y 62 años, todos eran clase social media. Los participantes tenían estudios universitarios completos e incompletos y secundario completo e incompleto.

Instrumentos. Estado de ánimo: Escala de Valoración del Estado de Ánimo (EVEA, Sanz, 2001). Es una escala de carácter situacional, evalúa cómo se siente la persona en ese momento, elaborada para detectar los cambios que se puedan operar en esos estados producto de cambios ambientales $y$ 
contextuales. Tiene 16 ítems, por lo que puede aplicarse en menos de dos minutos. Cada ítem tiene la misma construcción; empiezan con las palabras "me siento" y continúan con un adjetivo que representa un estado de ánimo (por ej., "me siento triste", "me siento alegre"); cada uno de ellos tiene una escala gráfica de tipo Likert de 11 puntos (de 0 a 10), flanqueadas por las palabras "nada" (0) a "mucho" (10). La EVEA evalúa cuatro estados de ánimo: 1) ansiedad, 2) ira-hostilidad, 3) tristeza-depresión y 4) alegría. Cada estado de ánimo viene representado por cuatro ítems con diferentes adjetivos los cuales definen una subescala, y todos están formulados en la misma dirección. Cada ítem se valora de 0 a 10 puntos en función del valor escogido por la persona evaluada y, tras sumar directamente la puntuación de los cuatro adjetivos correspondientes a cada subescala y dividir la suma por 4 , se obtienen cuatro puntuaciones entre 0 y 10 que cuantifican los estados de ánimo en el momento en que se administró el instrumento. Una puntuación más alta en las subescalas de la EVEA indica, un mayor nivel del estado de ánimo que representa. Del PinoSedeño, Peñate \& Bethencourt (2010) validaron el EVEA con el PANAS; hallando que EVEA tiene una fuerte consistencia interna, una estructura factorial robusta, una conformación de acuerdo con las cuatro variables previstas y una alta correlación con el PANAS. Las cuatro subescalas presentaron elevados índices de consistencia interna, con un coeficiente Alfa de Cronbach entre 0,88 y 0,93 (Sanz, 2001); el de la presente muestra presentó un coeficiente Alfa de Cronbach entre 0.75 a 0.80 .

-Encuesta sociodemográfica: indagó sobre edad, sexo, lugar de residencia, personas con quien convive, estudios realizados y profesión u ocupación.

-Procedimiento. Los participantes, fueron invitados a un almuerzo. Previamente se les preguntaron si querían participar en una investigación sobre el estado de ánimo en jóvenes y adultos. Todos aceptaron, por lo cual, después del consentimiento informado, se entregó el EVEA y la encuesta sociodemográfica. Luego comenzó el almuerzo (asado con bebidas sin alcohol y vino) y el grupo se quedó se quedó conversando de diferentes temas alrededor de 3 horas. Antes de retirarse se les volvió a entregar el EVEA a cada participante. Al mes siguiente, se convocó al mismo grupo para otro almuerzo similar. Cuando llegaron se les entregó nuevamente el EVEA a cada participante. Al finalizar el almuerzo y luego de una sobremesa más breve que la anterior, se los invitó a jugar. Al finalizar con los juegos, antes de retirarse se les volvió a entregar el EVEA a cada participante. Pasaron 3 horas aproximadamente luego del almuerzo (Ver Tabla 1 con el resumen del diseño experimental)

Tabla 1

Síntesis del diseño utilizado

\begin{tabular}{lllcllll}
\hline & $\mathbf{1}^{\mathbf{0}}$ encuentro & & Inter & \multicolumn{3}{c}{$\mathbf{2}^{\mathbf{2}}$ encuentro } \\
\cline { 1 - 1 } Pretest & Almuerzo & Postest & 30 & días & Pretest & Almuerzo & Postest \\
& sin juegos & & & & con juegos & \\
Sí & & Sí & & Sí & & Sí \\
EVEA & & EVEA & & EVEA & & EVEA \\
\hline
\end{tabular}


Los juegos realizados fueron de creatividad, de lectura y escritura: el Taboo y el Cadáver exquisito. El Taboo se juega con dos equipos, con cartas y por tiempo, donde cada jugador debe describir una palabra $\mathrm{X}$ sin mencionar 5 palabras taboo, o sea, prohibidas, que derivan en la palabra $X$. Por ejemplo, si la palabra X a describir es "helicóptero", y las palabras taboo son "piloto", "volar", "cielo", "hélice", "helipuerto", el juego consiste en cómo describir, en la menor cantidad de tiempo, la palabra helicóptero sin mencionar las 5 palabras taboo. Los equipos se sientan intercalados, de modo que puedan ser controlados por cada integrante del equipo contrario de no mencionar las palabras taboo. El equipo que acierta la mayor cantidad de palabras en 2 minutos de tiempo, por ronda, es el ganador. El Cadáver exquisito consiste en que cada participante escriba una frase libremente y la pase al jugador de al lado. Éste luego de leerla, escribe otra frase continuándola, tapa la frase anterior y la entrega al compañero de al lado. Este último sólo leerá la última oración y escribirá otra continuando su sentido, y así sucesivamente. Al final de la página, se hará un cierre de la historia inventando un final. Luego se leen todas las historias.

\section{Análisis de los resultados}

Se utilizó el programa de estadística SPSS versión 20. Una vez recolectados los instrumentos, se codificaron las respuestas, obteniendo las puntuaciones de cada sujeto y de la muestra en relación con cada una de las subescalas. Para el análisis de los resultados, primero se realizó la prueba de normalidad. Se estableció como criterio de significación un alfa de $\mathrm{p}<.05$. El tamaño del efecto se calculó con $\mathrm{r}=\mathrm{Z}$ / raíz cuadrada de $\mathrm{N}$.

\section{Resultados}

La Tabla 2 muestra los resultados de la prueba de normalidad. Se observa que las variables cumplen con los criterios de normalidad por lo que se utilizó la prueba $\mathrm{T}$ Student para muestras relacionadas.

\section{Tabla 2}

\section{Prueba de normalidad}

\begin{tabular}{|c|c|c|c|c|c|c|}
\hline & Kolmogoro & mil & & Shapiro-W & & \\
\hline & Estadístico & $\mathrm{gl}$ & Sig. & Estadístico & $\mathrm{gl}$ & Sig. \\
\hline $\begin{array}{l}\text { Estado de Ánimo - } \\
\text { Antes }\end{array}$ & ,156 & 10 & ,200* & ,971 & 10 & ,897 \\
\hline $\begin{array}{l}\text { Estado de Ánimo - } \\
\text { Después }\end{array}$ &, 183 & 10 &, $200^{*}$ & ,917 & 10 & ,335 \\
\hline
\end{tabular}


Las Figuras 1 y 2 muestran los promedios de la EVEA antes y después en la condición sin juegos y en la con juegos respectivamente. En ambos almuerzos se observa una disminución de la tristeza, ansiedad, hostilidad y un aumento de la alegría. Además, esta última en ambas condiciones y tanto antes como después tiene puntajes mayores que las demás emociones.

En primer lugar, se analizaron los puntajes de la EVEA antes y después de las condiciones experimentales por separado. En ninguna de las variables aplicadas antes y después del almuerzo se obtuvieron diferencias significativas $(\mathrm{p}>0.05)$.

En segundo lugar, los datos se analizaron antes y después de cada condición. En la condición sin juego, la ansiedad fue significativamente menor después del almuerzo con alto tamaño del efecto: $\mathrm{T}(9)=$ $2.44, \mathrm{p}<.04, \mathrm{r}=0.77$. Las demás emociones no obtuvieron diferencias significativas entre antes y después del almuerzo ( $\mathrm{p}>.05$ ).

En la condición con juego en todas las variables hubo diferencias significativas entre antes y después del encuentro con un alto tamaño del efecto: Tristeza: $\mathrm{T}(9)=2.24$, $\mathrm{p}<.05, \mathrm{r}=0.71$; Ansiedad: $\mathrm{T}(9)=3.19, \mathrm{p}<.01$, $\mathrm{r}=1$; Ira y hostilidad: $\mathrm{T}(9)=3.20, \mathrm{p}<.01, \mathrm{r}=$ 1 y Alegría: $\mathrm{T}(9)=3.09, \mathrm{p}<.01, \mathrm{r}=1$. Finalmente, la alegría obtuvo puntaje significativamente mayor en comparación con todos los demás puntajes de las emociones evaluadas (<.0001) en ambas condiciones experimentale.
Figuras 1 y 2

Promedio del EVEA en función de antes y después del tipo de encuentro
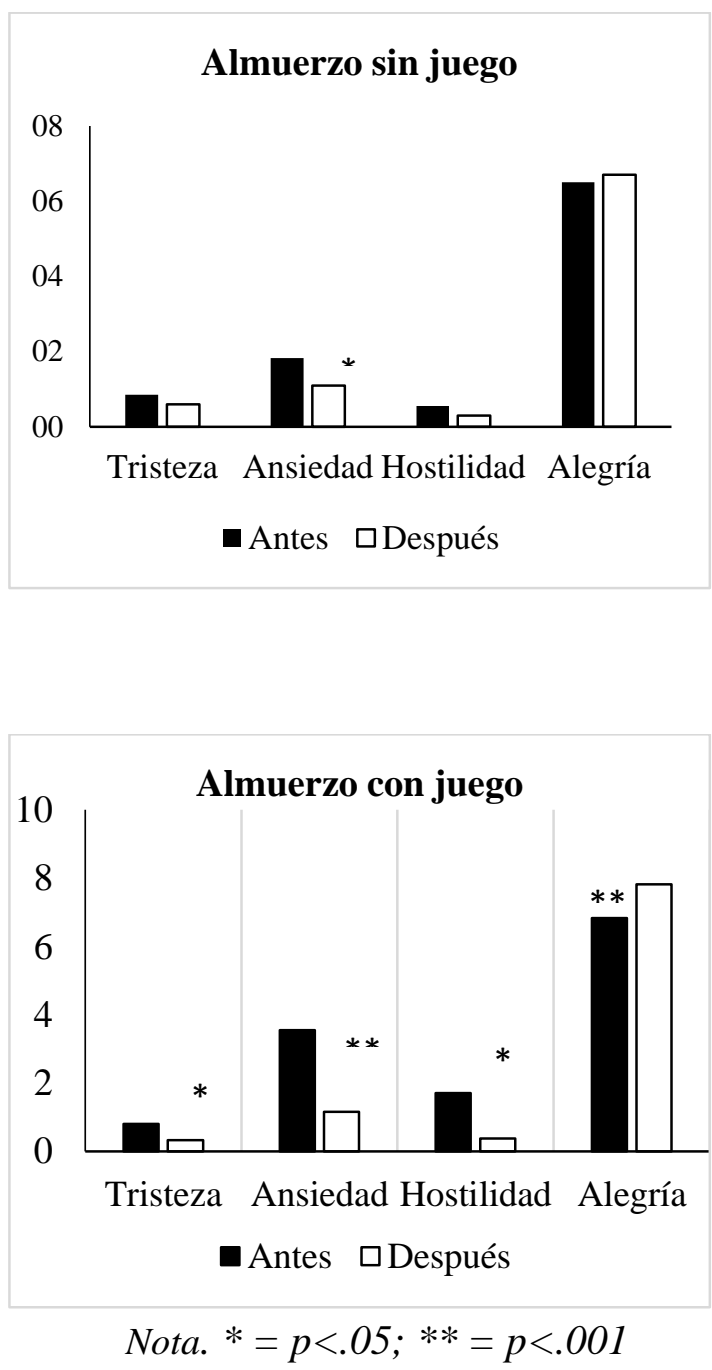

\section{Discusión y conclusiones}

El juego acompaña siempre al hombre a lo largo de toda su vida, aunque su forma evolucionará con su maduración y desarrollo. Inicialmente el niño se encuentra desbordado por una gran acumulación de energía sobrante y una de las maneras de descargarla es a través del juego, aunque se divierten con ellos. Además, cumple una función de aprendizaje 
porque suelen jugar imitando conductas de adultos, como cocinar, ir a trabajar, jugar a las visitas, etc. El adulto juega para disfrutar, para ocupar su tiempo libre, para desarrollarse con otras personas o para liberar las tensiones acumuladas a lo largo de la jornada laboral, entre otros motivos (Velásquez, 2014).

Las investigaciones sobre juegos en adultos mostraron eficacia en el desarrollo de funciones cognitivas y motrices y disminución de la depresión, aunque son escasas las investigaciones con diseños experimentales y la mayoría se realizaron con adultos mayores o enfermos.

Los resultados principales de esta investigación mostraron que tanto en un almuerzo con o sin juegos la tristeza, la ansiedad y la hostilidad disminuyeron y la alegría fue mayor después de un almuerzo. Además, la alegría obtuvo altos puntajes en ambas condiciones. Sin embargo, solamente la ansiedad disminuyó significativamente en ambas condiciones. En cambio, en la condición de almuerzo con juegos de cartas y de lectura y escritura después de una breve sobremesa, todas las diferencias fueron significativas y con un robusto tamaño del efecto comparadas con las medidas tomadas antes del almuerzo: disminuyó significativamente la tristeza, la ansiedad y la hostilidad y aumentó significativamente la alegría.

Estos datos muestran, en primer lugar, que en los encuentros sociales la alegría predomina en los estados de ánimo de los participantes respecto de las otras emociones negativas desde el principio de la reunión, probablemente debido a la expectativa de pasar un rato agradable con amigos, y que la ansiedad disminuye al final del encuentro.
Esto está apoyado por las numerosas investigaciones que muestran que, en los animales sociales, la pertenencia a grupos sociales es un potente reforzador positivo que provoca placer y bienestar (e.g., Baumeister \& Leary 1995). En segundo lugar, la inclusión de juegos durante la reunión aumentó el valor reforzante de la reunión social más que una sobremesa de conversación; indica que los juegos potencian aún más el bienestar de las personas en las reuniones sociales.

Estos resultados apoyan la hipótesis propuesta y muestran evidencia que los juegos de cartas y de escritura y lectura mejoran el estado de ánimo y es similar a lo que encontraron otros autores con adultos mayores y personas con deterioro cognitivo (ej. Noda \& cols.,2019). En este sentido este estudio comprueba experimentalmente lo que intuitivamente se conoce, y es que una manera para inducir emociones positivas en adultos son los juegos; brinda otro apoyo empírico sobre la importancia de estimular el juego en todas las etapas evolutivas, y pueden ser importantes recursos tanto para la promoción de la salud emocional y el bienestar psicológico, como en ámbitos educacionales y de rehabilitación.

Como toda investigación, ésta tiene sus limitaciones: la muestra fue reducida, el grupo homogéneo en su nivel cultural y no se pudo realizar un contrabalanceo en las condiciones experimentales, aunque el intervalo entre las dos condiciones pudieron garantizar que no hubo efecto del orden de presentación. Habría que replicar los resultados con una muestra más amplia y evaluar distintos juegos teniendo en cuenta las preferencias de los participantes. Además, se deberían realizar futuras investigaciones para corroborar estos resultados y evaluar el 
alcance de los cambios emocionales que puede producir el uso del juego de modo reiterado. Además, se abren una serie de interrogantes a ser estudiados en el futuro, entre ellos ise podrían generar a través del juego nuevas formas de pensamiento más flexibles, creativas y menos rígidas, con las cuales

\section{Referencias}

Aciego, R., García, L. \& Betancort, M. (2016). Efectos del método de entrenamiento en ajedrez, entrenamiento táctico versus formación integral, en las competencias cognitivas y sociopersonales de los escolares. Universitas Psychologica, 15(1), 1526.

Bilalic', M., Langner, R., Erb, M., \& Groud, W. (2010). Mechanisms and neural basis of object and pattern recognition: A study with chess experts. Journal of Experimental Psychology: General, 138, 728-742. http://dx.doi.org/10.1037/a0020756

Bilalic', M., McLeod, P., \& Gobet, F. (2009). Specialization effect and its influence on memory and problem solving in expert chess players. Cognitive Science, 33, 1117-1143. http://dx.doi.org/10.1111/j.15516709.2009.01030.x

Campitelli, G., Gobet, F. \& Parker, A. (2005) Estructura y familiaridad del estímulo: un estudio de la memoria en jugadores de ajedrez con resonancia magnética funcional. Span Journal of psychology, 8(2), 238-45.

Carrasco Zumba, L. \& Cuenca Quito, E. (2015). La práctica del ajedrez en elaborar respuestas novedosas que permitan ver un problema más objetivamente y encontrar una solución más efectiva?, ¿participar de juegos regularmente provocará un mejor estado de ánimo duradero? ¿se podrán aplicar estos juegos en ámbitos escolares?

personas de la tercera edad del Centro de Jubilación Activa del IESS, efectos y estudio; Cuenca 2015. (Trabajo de grado). Universidad de Cuenca, Facultad de Filosofía, Letras y Ciencias de la Educación, Ecuador.

Charness, N., Tuffiash, M., \& Krampe, R. (2005). The role of deliberate practice in chess expertise. Applied Cognitive Psychology, 19, 151-165. http://dx.doi.org/10.1002/acp.1106

Chase, W. G., \& Simon, H. A. (1973). Perception in chess. Cognitive Psychology, 4, 55-81. http://dx.doi.org/10.1016/00100285(73)90004-2

De Groot, A.D. (1946). Het denken van den Schaker, een experimenteelpsychologische studie [The thinking of the chess player: an experimental psychological study]. Doctoral Thesis, University of Amsterdam. Amsterdam, Holland. http://www.dbnl.org/tekst/groo004de nk01_01/groo004denk01_01.pdf

De Groot, A.D. (1965). Thought and choice in chess. The Hague, The Netherlands: Mouton \& Co Publishers. 
Del Pino-Sedeño, T., Peñate, W. \& Bethencourt, J. M. (2010). La escala de valoración del estado de ánimo (EVEA): análisis de la estructura factorial y de la capacidad para detectar cambios en estados de ánimo. Análisis y Modificación de Conducta, 36 (153-154), 19-32.

Duan, X.; He, Sh.; Liao, W.; Liang, D.; Qiu, L.; Wei, L., Chen, H. (2012). Reduced caudate volume and enhanced striatalDMN integration in chess experts. NeuroImage $\quad 60, \quad 1280-1286$. http://dx.doi.org/10.1016/j.neuroimag e.2012.01.047.

Gobet, F. \& Campitelli, G. (2007). The role of domain-specific practice, handedness, and starting age in chess. Developmental Psychology, 43(1), 159-72. http://dx.doi.org/10.1037/00121649.43.1.159

Gobet, F., \& Simon, H. A. (1996). Templates in chess memory: A mechanism for recalling several boards. Cognitive Psychology, 31, 1-40. http://dx.doi.org/10.1006/cogp.1996.0 011

Gobet, F., \& Simon, H. A. (1998). Expert chess memory: Revisiting the chunking hypothesis. Memory, 6, 225-255.

http://dx.doi.org/10.1080/741942359

Hernández, P., \& Rodríguez, H. (2006). Success in chess mediated by mental molds. Psicothema, 18, 704-710.
Hopelab (2019). Luchando contra el cáncer con videojuegos. https://www.remission2.org/\#research

Kato, P., Cole, S., Bradlyn, A. y Pollock, B. (2008). Un videojuego mejora los resultados conductuales en adolescentes y adultos jóvenes con cáncer: un ensayo aleatorio. Revista Pediatrics, 122(2), 305-317. https://pediatrics.aappublications.org/ content/122/2/e305

Krawczyk, D. C., Boggan, A. L., McClelland, M. M., \& Bartlett, J. C. (2011). The neural organization of perception in chess experts. Neuroscience Letters, 499,

64-69. http://dx.doi.org/10.1016/j.neulet.201 1.05 .033

Linares Cervantes, R. (2015). La eficacia del juego y la creatividad para la integración social de los inmigrantes (Tesis doctoral). https://eprints.ucm.es/38959/1/T3773 6.pdf

Linhares, A. (2005). An active symbols theory of chess intuition. Minds and Machines, 15, 131-181. http://dx.doi.org/10.1007/s11023005-5045-7

Linhares, A., \& Freitas, A. E. (2010). Questioning Chase and Simon's (1973) "perception in chess": The "experience recognition" hypothesis. New Ideas in Psychology, 28, 64-78. http://dx.doi.org/10.1016/j.newideaps ych.2009.07.008

Linhares, A., Freitas, A.E., Mendes, A., \& Silva, J.S. (2012). Entanglement of 
perception and reasoning in the combinatorial game of chess: Differential errors of strategic reconstruction. Cognitive Systems Research, 13, 72-86. http://dx.doi.org/10.1016/j.cogsys.20 10.12 .006

Perandones Serrano, E. (2010). Videojuegos para la salud. VI Curso de Comunicación y Salud. Nuevos escenarios y tendencias en tiempos de crisis. Madrid: Universidad Complutense de Madrid.

Pérez-Sánchez, R. \& Torres, D. (2014). Intensidad de demanda de los videojuegos y su efecto sobre el estado de ánimo y la activación percibida. Universitas Psychologica, 13(4), 1489-1502.

Romero, K. y Alonso, L. (2004). Arte y juego en las Aulas Hospitalarias: una experiencia en el Hospital Universitario de Los Andes. Universidad de Los Andes, Mérida, Venezuela.
Sanz, J. (2001). Un instrumento para evaluar la eficacia de los procedimientos de inducción de estado de ánimo: "La Escala de Valoración del Estado de Ánimo" (EVEA). Análisis y Modificación de Conducta, 27, 71100.

Vega, J., Buz, J. \& Bueno, B. (2002). Niveles de actividad y participación social en las personas mayores de 60 años. Revista Interuniversitaria de Formación del Profesorado, 45, 3353.

Velasquez, M. (1 de julio de 2014). Juegos digitales. [Entrada de blog]. http://majuveva.blogspot.com.ar/2014 /07/juegos-digitales.html

Zhang, F. \& Kaufman, D. (2016) Physical and Cognitive Impacts of Digital Games on Older Adults: A Meta-Analytic Review. Journal of Applied Gerontology, 35(11), 1189-1210 http://dx.doi.org/10.1177/073346481 4566678 